\title{
Trends in Astrobiology - Comets, Pluto and beyond
}

Chandra Wickramasinghe $\mathbf{N}^{1,2,3}$

${ }^{1}$ Buckingham Centre for Astrobiology (BCAB), Buckingham University, UK

${ }^{2}$ Institute for the Study of Panspermia and Astroeconomics, Gifu, Japan

${ }^{3}$ University of Peradeniya, Peradeniya, Sri Lanka

${ }^{*}$ Corresponding author: Wickramasinghe NC, Buckingham Centre for Astrobiology (BCAB), Buckingham University, UK, Tel: +44-777-838-9243; E-mail: ncwick@gmail.com

Rec date: July 14, 2015; Acc date: July 16, 2015; Pub date: July 20, 2015

Copyright: (C) 2015 Chandra Wickramasinghe N. This is an open-access article distributed under the terms of the Creative Commons Attribution License, which permits unrestricted use, distribution, and reproduction in any medium, provided the original author and source are credited.

\section{Editorial}

There is now a large body of evidence showing the widespread occurrence of life-related organic molecules in the Universe [1-3]. There is also clear evidence pointing to life processes taking place within comets when they approach the sun $[4,5]$. The data obtained recently from the Rosetta Mission to comet 67P/C-G could not, in the view of the writer, be explained easily except on the basis of on-going subsurface microbiology [6,7] (Figure 1).

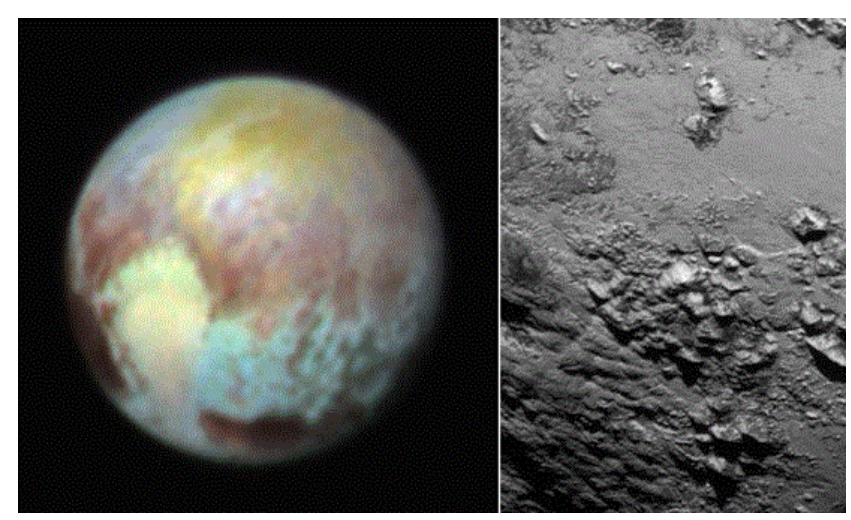

Figure 1: Pluto (New Horizons, 13th, 14th July 2015, Courtesy NASA). Left: Enhanced colour, showing regions probably containing organics, Right: Closer-up image of surface, showing rugged terrain

The New Horizons spacecraft that swung past the dwarf planet Pluto on 14 July 2015 is beginning to yield data that may also be pointing in the same direction. Pluto is the largest member of a swarm of so-called Kuiper-belt objects that occupy the frozen outer reaches of the solar system, beyond the orbit of Neptune. With a predominantly comet-like icy composition they could well turn out to be a major reservoir of microbial life in the outer solar system. Although the temperature near the surface of Pluto is well below the limit for microbial replication, interior domains a few hundred kilometres below the surface, heated by radioactive decays, could maintain water in liquid form for billions of years and thus be able to support active microbial life. On this hypothesis we can expect to see evidence of "cryo-volcanism" constantly turning over the surface and bringing up surface layers containing methane and organic pigments that would point to microbiology. The red patches on Pluto and its moon Charon have been explained by NASA as possibly arising from nitrogen particles. But the red as well as the green and yellow patches can be easily explained on the basis of microbiology. There will be a lot more data to come from the New Horizons mission and doubtless every effort will be made to explain all of them in terms of non-biological processes. The trend of the observations, however, seems clear. In the writer's view it is high time to give up the struggle to reject biology and be guided by facts rather than prejudice.

Astrobiology's remit should be extended forthwith from the omnipresence of the chemical building blocks of life to the widespread cosmic distribution of life itself. Panspermia as a working hypothesis should rightly be at the top of the astrobiologist's agenda. Instead it is still widely dismissed as a "way out" idea - an extraordinary idea for which the requisite extraordinary evidence is claimed wrongly to be lacking.

In view of the rapid convergence of supportive evidence it is surprising that a paradigm shift from Earth-centred biology to life as a cosmic phenomenon has not already happened. Long after the Earth lost its privileged status as the physical centre of the world, following the discoveries of Galileo, Copernicus and Kepler, our planet continues to occupy centre stage in relation life. Change is unquestionably overdue.

The Vatican's recent pronouncements [8] on extraterrestrial life (attributed to Papal Astronomer Brother Guy Consolmagno) are to be welcomed. Even though the much-hyped Papal/Vatican remarks concern extraterrestrial intelligence and thus goes outside the strict remit of astrobiology it is nevertheless a step in the right direction. Whilst most of the world's scientific establishments appear to be turning away from accepting the evidence that supports extraterrestrial life by finding any excuse to devalue or denigrate it, the most conservative of religious establishments appears to be displaying a remarkably progressive trend. This is good news particularly for an institution that had once burnt Giodarno Bruno at the stake for pronouncing a multiplicity of inhabited alien worlds, and convicted Gallileo Gallilei for heresy.

With the estimated total of exoplanets in the galaxy now exceeding 100 billion [9] and the widespread cosmic occurrence of complex organic molecules, commonsense alone should dictate that biology is commonplace and that life is a truly cosmic phenomenon. Whilst the first origin of life from non-lie still remains an unsolved mystery, what astrobiology and astronomy informs us is that once life starts its persistence and spread is inevitable and unstoppable. These new trends are a fitting tribute of the late Sir Fred Hoyle whose birth centenary fell on 24 June this year. 
Citation: Chandra Wickramasinghe N (2015) Trends in Astrobiology - Comets, Pluto and beyond. Astrobiol Outreach 3: 109e. doi: $10.4172 / 2332-2519.1000109 \mathrm{e}$

Page 2 of 2

\section{References}

1. Wickramasinghe NC (2010) The astrobiological case for our cosmic ancestry. International Journal of Astrobiology 9: 119-129

2. Wickramasinghe NC, Tokoro G (2014) Life as a Cosmic Phenomenon: The Socio-Economic Control of a Scientific Paradigm. Astrobiol Outreach 2: 113

3. Wickramasinghe NC, Tokoro G (2014) Life as a Cosmic Phenomenon 2.The Panspermia Trajectory of Homo sapiens. Astrobiol Outreach 2: 115

4. Wickramasinghe J, Wickramasinghe C, Napier W (2011) Comets and the Origins of Life. World Scientific Press Singapore.

5. Wickramasinghe NC (2014) The Search for Our Cosmic Ancestry. World Scientific Press, Singapore.
6. Wallis MK, Wickramasinghe NC (2015) Rosetta Images of Comet 67P/ Churyumov-Gerasimenko: Inferences from Its Terrain and Structure. Astrobiol Outreach 3: 127

7. Wickramasinghe NC, Wainwright M, Smith WE, Tokoro G, Al Mufti S, et al. (2015) Rosetta Studies of Comet 67P/Churyumov-Gerasimenko: Prospects for Establishing Cometary Biology. Astrobiol Outreach 3: 126

8. http://www.ibtimes.co.uk/popes-astronomer-guy-consolmagno-saysaliens-exist-1467400

9. Wickramasinghe NC and Smith W (2014) Convergence to panspermia. Hypothesis 12: e9 